MATHEMATICS OF COMPUTATION

Volume 81, Number 278, April 2012, Pages 1053-1061

S $0025-5718(2011) 02537-8$

Article electronically published on August 25, 2011

\title{
AN IMPROVED UPPER BOUND FOR THE ARGUMENT OF THE RIEMANN ZETA-FUNCTION ON THE CRITICAL LINE
}

\author{
TIMOTHY TRUDGIAN
}

\begin{abstract}
This paper concerns the function $S(t)$, the argument of the Riemann zeta-function along the critical line. Improving on the method of Backlund, and taking into account the refinements of Rosser and McCurley it is proved that for sufficiently large $t$,

$$
|S(t)| \leq 0.1013 \log t
$$

Theorem 2 makes the above result explicit, viz. it enables one to select values of $a$ and $b$ such that, for $t>t_{0}$,
\end{abstract}

$$
|S(t)| \leq a+b \log t
$$

\section{INTRODUCTION}

This paper pertains to the argument of $\zeta(s)$, the Riemann zeta-function, along the critical line $s=\frac{1}{2}+i t$. Whenever $t$ does not coincide with an ordinate of a zero of $\zeta(\sigma+i t)$ one defines the function $S(t)$ as

$$
S(t)=\pi^{-1} \arg \zeta\left(\frac{1}{2}+i t\right),
$$

where the argument is determined via continuous variation along the straight lines connecting $2,2+i t$ and $\frac{1}{2}+i t$, with $S(0)=0$. If $t$ is such that $\zeta(\sigma+i t)=0$, then define $S(t)=\frac{1}{2} \lim _{\epsilon \rightarrow 0}\{S(t-\epsilon)+S(t+\epsilon)\}$. Without assuming unproven conjectures (for example the Riemann or Lindelöf hypotheses) the classic estimate of von Mangoldt, $S(t)=O(\log t)$, has never been improved, except by reducing the size of the implied constant. Backlund [1] showed that, for $t \geq 200$,

$$
|S(t)| \leq 0.137 \log t+0.445 \log \log t+4.35,
$$

where the lower order terms were improved by Rosser [9], who showed that, for $t \geq 1467$,

$$
|S(t)| \leq 0.137 \log t+0.443 \log \log t+1.588,
$$

and a computational check shows that this remains valid for all $t \geq 3$. Such explicit results are useful when estimating sums over the zeroes of $\zeta(s)$; see, e.g. [4, 10.

The main idea of Backlund's Method is to count the number of zeroes of $\Re \zeta(\sigma+i t)$ on the line segment $\left[\frac{1}{2}+i t, 1+\eta+i t\right]$ where $0<\eta \leq \frac{1}{2}$. Suppose there are $n$ such zeroes, labelled $a_{1}, \ldots, a_{n}$. These zeroes partition the line segment $\left[\frac{1}{2}+i t, 1+\eta+i t\right]$ into $n+1$ intervals. On the interior of each interval, $\arg \zeta(s)$ can change by at most

Received by the editor October 21, 2010 and, in revised form, February 23, 2011.

2010 Mathematics Subject Classification. Primary 11M06; Secondary 11M26.

Key words and phrases. Riemann zeta-function, convexity estimate.

(C)2011 American Mathematical Society 
$\pi$, since by construction, $\Re \zeta(s)$ is non-zero on each interior. Thus, as $\sigma$ varies from $\frac{1}{2}$ to $1+\eta$, then

$$
|\Delta \arg \zeta(s)| \leq(n+1) \pi .
$$

One proceeds to bound $n$ from above using Jensen's formula on the function

$$
f(s)=\frac{1}{2}\left\{\zeta(s+i t)^{N}+\zeta(s-i t)^{N}\right\},
$$

for $N$ a natural number 1 ; thus $f(\sigma)=\Re \zeta(\sigma+i t)^{N}$. There are two ways to proceed.

Method $\mathcal{A}$ takes account of all the zeroes contained in a circle of radius $r\left(\frac{1}{2}+\eta\right)$, centered at $s=1+\eta+i t$, for some $r \in(1,2]$. McCurley [6] follows this line of attack, with $r=2$. Contrarily, method $\mathcal{B}$ makes use of a clever observation by Backlund, henceforth called 'Backlund's trick'.

For any $\delta \in\left[0, \frac{1}{2}+\eta\right)$, let $\Delta_{1} \arg \zeta(s)$ denote the change in the argument of $\zeta(s)$ as $\sigma$ varies from $\frac{1}{2}$ to $\frac{1}{2}+\delta$. Similarly, $\Delta_{2} \arg \zeta(s)$ is the change in argument as $\sigma$ varies from $\frac{1}{2}$ to $\frac{1}{2}-\delta$. By estimating the change in the argument of $\chi(s)$, where

$$
\zeta(s)=\chi(s) \zeta(1-s)=\pi^{s-\frac{1}{2}} \frac{\Gamma\left(\frac{1}{2}-\frac{1}{2} s\right)}{\Gamma\left(\frac{1}{2} s\right)} \zeta(1-s),
$$

(see, e.g., [11, Ch. II]) Backlund [pp. 355-357, op. cit.] was able to show that for $t>1$,

$$
\left|\Delta_{1} \arg \zeta(s)+\Delta_{2} \arg \zeta(s)\right| \leq \frac{8}{t} .
$$

It follows that there are at least 2$]-2$ zeroes of $\Re \zeta(s)$ on the line segment $[-\eta+$ $\left.i t, \frac{1}{2}+i t\right]$, and so at least $2 n-2$ zeroes of $\Re \zeta(s)$ for $\sigma \in[-\eta, 1+\eta]$. So one uses Jensen's formula, with a circle of radius $1+2 \eta$, centered at $s=1+\eta+i t$. McCurley's argument 3 works here as well, and gives [Thm 2.1, op. cit.]

$$
|S(t)| \leq 0.115 \log t
$$

for sufficiently large $t$.

The advantage of $\mathcal{B}$ is that one gets ' 2 -for-the-price-of- 1 ' in terms of the number of zeroes of $f(s)$. But the drawback is that one must estimate $|\zeta(s)|$ over the strip $-\eta \leq \sigma \leq 1+\eta$. With $\mathcal{A}$ one begins with fewer zeroes, but for a suitably small $r$, the incursion into the strip $\sigma \leq \frac{1}{2}$ is minimal. This is indeed an amelioration since, by convexity, $|\zeta(s)|$ grows much more quickly to the left of the line $\sigma=\frac{1}{2}$. Method $\mathcal{A}$ is outlined in [11, Ch. XIII, §9].

It must be noted that any detriment from using $\mathcal{B}$ is nullified if one uses the convexity bound $\left|\zeta\left(\frac{1}{2}+i t\right)\right| \ll t^{1 / 4}$. Thus, if method $\mathcal{A}$ is to be of any use, one must know the value of the constant $K$ for which $\left|\zeta\left(\frac{1}{2}+i t\right)\right| \leq K t^{\theta}$ where $\theta<\frac{1}{4}$. Cheng and Graham [2] have shown that

$$
\left|\zeta\left(\frac{1}{2}+i t\right)\right| \leq 3 t^{\frac{1}{6}} \log t
$$

for $t>e$, and this will be used in 4.1

The remainder of the paper sets out to prove

\footnotetext{
${ }^{1}$ Backlund has $N=1$. The introduction of the number $N$ and the passing through a sequence of $N$ s, tending to infinity is due to Rosser. The advantages of this will be made plain on p. 1056

${ }^{2}$ Alternatively, for large enough $t$ there are at least $n$ zeroes of $\Re \zeta(s)$. This matters little, especially in light of the improvements given by Rosser given on p. 1056

${ }^{3}$ McCurley considers Dirichlet $L$-functions, whence he is unable to make use of Backlund's trick. Also, he considers $N(t)$ to be those zeroes with imaginary part $\gamma \in[-t, t]$. Thus the upper bound in (1.3) is one quarter of that in 6].
} 
Theorem 1 (via Method $\mathcal{B}$ ). If $t>t_{0}>e$, then

$$
|S(t)| \leq 1.998+0.17 \log t .
$$

It should be noted that the theorem is valid for all $t>t_{0}>e$, and the particular choice of coefficients minimises the right-side of (1.5) when $t_{0}=10^{10}$. Better bounds for larger values of $t_{0}$ are calculable from $\$ 5$. The value of the coefficient of $\log t$ can be diminished further, but the limitations of the theorem show that it cannot be taken to be less than 0.1027. Any diminution in this coefficient is at the expense of increasing the constant term.

This paper can be considered a sequel to my paper on Turing's Method [12, and indeed many of the calculations involving convexity estimates for bounds on $\left|\zeta\left(\frac{1}{2}+i t\right)\right|$ are similar.

\section{The Requisites for BaCKLund's Method}

The opening gambits of Backlund and McCurley are essentially the same. One writes

$$
\xi(s)=\frac{1}{2} s(s-1) \pi^{-\frac{1}{2} s} \Gamma\left(\frac{1}{2} s\right) \zeta(s),
$$

where $\xi(s)$ is an entire function, whose zeroes coincide with the non-trivial zeroes of $\zeta(s)$. If one writes $N(t)$ as the number of complex zeroes of $\zeta(s)$ with imaginary part $\gamma \in[0, t]$, then it follows from Cauchy's theorem, the functional equation and the reflection principle that $4 \pi N(t)=4 \Delta_{R} \arg \xi(s)$, where $R$ is the pair of lines connecting the points $1+\eta, 1+\eta+i t$ and $\frac{1}{2}+i t$. In calculating the change in argument of $\xi(s)$ one finds a main term and then the term corresponding to $\Delta_{R} \arg \zeta(s)$, which is $\pi S(t)$. The vertical piece is easily handled, since here, $|\arg \zeta(s)| \leq|\log \zeta(s)| \leq$ $\log \zeta(1+\eta)$. What remains is to estimate $\Delta_{h} \arg \zeta(s)$ : the change in argument of $\zeta(s)$ along the line segment $\left[1+\eta+i t, \frac{1}{2}+i t\right]$.

With $f(s)$ defined as in (1.1), it follows that

whence

$$
\left|\Delta_{h} \arg \zeta(s)\right|=\frac{1}{N}\left|\Delta_{h} \arg \zeta(s)\right|^{N} \leq \frac{(n+1) \pi}{N},
$$

$$
|S(t)| \leq \frac{2}{\pi} \log \zeta(1+\eta)+\frac{n+1}{N} .
$$

One can now produce an upper bound on $n$ courtesy of method $\mathcal{A}$ or $\mathcal{B}$. The proof below is valid for any $r \in(1,2]$ and the difference between the two methods will be plainly seen.

\section{Bounding $n$ USING Method $\mathcal{A}$}

For $r \in(1,2]$, Jensen's formula is applied to the function $f(s)$ on a circle with radius $r\left(\frac{1}{2}+\eta\right)$ centered at $s=1+\eta+i t$, to give

$$
\begin{aligned}
n \log r & \leq \frac{1}{2 \pi} \int_{-\pi / 2}^{3 \pi / 2} \log \left|f\left(1+\eta+r\left(\frac{1}{2}+\eta\right) e^{i \phi}\right)\right| d \phi-\log |f(1+\eta)| \\
& =I_{1}+I_{2}+I_{3}+I_{4}-\log |f(1+\eta)|,
\end{aligned}
$$

where $I_{1}$ covers $\phi \in\left[-\frac{\pi}{2}, \frac{\pi}{2}\right], I_{2}$ covers $\phi \in\left[\frac{\pi}{2}, \frac{\pi}{2}+\sin ^{-1} r^{-1}\right], I_{3}$ covers $\phi \in$ $\left[\frac{\pi}{2}+\sin ^{-1} r^{-1}, \frac{3 \pi}{2}-\sin ^{-1} r^{-1}\right]$ and $I_{4}$ covers $\phi \in\left[\frac{3 \pi}{2}-\sin ^{-1} r^{-1}, \frac{3 \pi}{2}\right]$.

\footnotetext{
${ }^{4}$ One can use Cauchy's theorem and the fact that $\arg \zeta(2)=\arg \zeta(1+\eta)=0$ to show that calculating $\Delta \arg \zeta(s)$ along the aforementioned lines agrees with the definition of $S(t)$.
} 
After this dividing of ranges of integration one notes that $\Re(s) \geq 1+\eta$ on $I_{1}$, whence one estimates $f(s)$ trivially, viz.

$$
\log |f(s)| \leq N \log \zeta(1+\eta) .
$$

On both $I_{2}$ and $I_{4}, \Re(s) \geq \frac{1}{2}$, so that it is only on $I_{3}$ that $-\eta \leq \Re(s) \leq \frac{1}{2}$, and this contribution diminishes as $r$ is taken closer and closer to unity.

To handle the $\log |f(1+\eta)|$ term, one makes use of the trick of Rosser. Write $\zeta(1+\eta+i t)=k e^{i \psi}$. Now choose a sequence of $N$ 's tending to infinity such that $N \psi$ tends to 0 modulo $2 \pi$, whence

$$
\lim _{N \rightarrow \infty} \frac{f(1+\eta)}{|\zeta(1+\eta+i t)|^{N}}=1 .
$$

Finally, for $\sigma>1$, one can consider the Euler product of $\zeta(s)$ to show that $|\zeta(s)| \geq$ $\frac{\zeta(2 \sigma)}{\zeta(\sigma)}$, whence the bound

$$
-\log |f(1+\eta)| \leq N \log \zeta(1+\eta) .
$$

The only terms in (3.1) left to estimate are $I_{2}$ and $I_{3}$; a bound for $I_{2}$ will serve as a bound for $I_{4}$. Explicit estimates of the growth of $|\zeta(\sigma+i t)|$ for $\sigma \in\left[-\eta, \frac{1}{2}\right]$ and for $\sigma \in\left[\frac{1}{2}, 1+\eta\right]$ are given in the following section.

\section{Preliminary Results}

An explicit version of the Phragmén-Lindelöf theorem is needed, as follows.

Lemma 1. Let $a, b, Q$ and $k$ be real numbers, and let $f(s)$ be regular analytic in the strip $-Q \leq a \leq \sigma \leq b$ and satisfy the growth condition

$$
|f(s)|<C \exp \left\{e^{k|t|}\right\},
$$

for a certain $C>0$ and for $0<k<\pi /(b-a)$. Also, assume that

$$
|f(s)| \leq \begin{cases}A|Q+s|^{\alpha} & \text { for } \Re(s)=a, \\ B|Q+s|^{\beta} & \text { for } \Re(s)=b\end{cases}
$$

with $\alpha \geq \beta$. Then throughout the strip $a \leq \sigma \leq b$ the following holds:

$$
|f(s)| \leq A^{(b-\sigma) /(b-a)} B^{(\sigma-a) /(b-a)}|Q+s|^{\alpha(b-\sigma) /(b-a)+\beta(\sigma-a) /(b-a)} .
$$

Proof. This is a result of Rademacher and can be found in [8, pp. 66-67].

In order to apply Lemma 1 one needs bounds on $|\zeta(s)|$ on each of the three lines: $\sigma=1+\eta, \quad \sigma=\frac{1}{2}$, and $\sigma=-\eta$. Trivially,

$$
|\zeta(1+\eta+i t)| \leq \zeta(1+\eta) .
$$

The bound of Cheng and Graham (1.4) may be used on the line $\sigma=\frac{1}{2}$. One can bound $|\zeta(-\eta+i t)|$ by using the functional equation (1.2), (4.1) and the following result due to Rademacher.

Lemma 2. For $-\frac{1}{2} \leq \sigma \leq \frac{1}{2}$,

$$
\left|\frac{\Gamma\left(\frac{1}{2}-\frac{1}{2} s\right)}{\Gamma\left(\frac{1}{2} s\right)}\right| \leq\left(\frac{|1+s|}{2}\right)^{\frac{1}{2}-\sigma} .
$$

Proof. See [7, p. 197]. 
It follows that

$$
|\zeta(-\eta+i t)| \leq\left(\frac{|s+1|}{2 \pi}\right)^{\frac{1}{2}+\eta} \zeta(1+\eta) .
$$

The following lemma contains two estimates on the growth of $|\zeta(s)|$ in strips on either side of the critical line.

Lemma 3. Suppose there exist constants $B$ and $\theta$ satisfying

$$
\left|\zeta\left(\frac{1}{2}+i t\right)\right| \leq B|s+1|^{\theta},
$$

for all $t$. For $\frac{1}{2} \leq \sigma \leq 1+\eta$ and $t>t_{0}>e$,

$$
|\zeta(s)| \leq\left\{C_{1}^{\theta(1+\eta-\sigma)+\frac{1}{2}+\eta} B^{1+\eta-\sigma} \log \zeta(1+\eta)^{\sigma-\frac{1}{2}} t^{\theta(1+\eta-\sigma)}\right\}^{1 /\left(\frac{1}{2}+\eta\right)}
$$

where

$$
C_{1}=\sqrt{1+\left(\frac{2+\eta}{t_{0}}\right)^{2}}
$$

Also, for $-\eta \leq \sigma \leq \frac{1}{2}$ and $t>t_{0}>e$,

$$
|\zeta(s)| \leq\left\{\left[\frac{\zeta(1+\eta)}{(2 \pi)^{\frac{1}{2}+\eta}}\right]^{\frac{1}{2}-\sigma} B^{\sigma+\eta}\left\{C_{2} t\right\}^{\left(\frac{1}{2}+\eta\right)\left(\frac{1}{2}-\sigma\right)+\theta(\sigma+\eta)}\right\}^{1 /\left(\frac{1}{2}+\eta\right)},
$$

where

$$
C_{2}=\sqrt{1+\frac{1}{t_{0}^{2}}}
$$

Proof. Equations (4.1) and (4.3) prove (4.4); equations (4.3) and (4.2) prove (4.5).

Equation (4.3) is present solely to give a suitable form of (1.4) in Lemma 11. To prove (4.4) take5, in Lemma 1, $f(s)=(s-1) \zeta(s), a=\frac{1}{2}, b=1+\eta, Q=1$, and use (4.3) and (4.1). The term $C_{1}$ springs from replacing $|s-1|$ with $|s+1|$.

To prove (4.5) take $f(s)=\zeta(s), a=-\eta, b=\frac{1}{2}, Q=1$, and use (4.2) and (4.3). The term $C_{2}$ is obtained by replacing $|s+1|$ with $t$.

4.1. The value of $B$. To arrive at (4.3) consider (1.4), viz.

$$
\left|\zeta\left(\frac{1}{2}+i t\right)\right| \leq 3 t^{1 / 6} \log t \leq 3|s+1|^{1 / 6} \log t,
$$

for $t>e$. To accommodate the $\log t$ term, note that one can choose a small $\delta$ and hence find a (large) $A_{0}=A_{0}\left(\delta, t_{0}\right)$ such that $\log t \leq A_{0} t^{\delta} \leq A_{0}|s+1|^{\delta}$, for $t \geq t_{0}$. Since the function $\log t / t^{\delta}$ never exceeds $(\delta e)^{-1}$, it follows that for all $t>e$,

$$
\left|\zeta\left(\frac{1}{2}+i t\right)\right| \leq \frac{3}{\delta e}|s+1|^{1 / 6+\delta},
$$

and a computational check shows the above to be valid for all $t \geq 0$. Thus we may take

$$
B=B(\delta)=\frac{3}{\delta e}
$$

\footnotetext{
${ }^{5}$ Note that Lemma 1 cannot be applied directly to $\zeta(s)$ owing to the pole at $s=1$.
} 
However, this presupposes that at a reasonable height for computation one wishes to use the bound (1.4) as opposed to the 'ordinary' convexity estimate

$$
\left|\zeta\left(\frac{1}{2}+i t\right)\right| \leq 2.53|1+s|^{\frac{1}{4}}
$$

which can be deduced from that in [5. It is clear that as $t$ increases one should prefer (1.4) to (4.7) but, as will be shown in the next section, this preference is not immutable, particularly for modest values of $t$. Indeed, the dependence of $B$ on $\delta$ is the primary source of frustration in seeking an improvement to Backlund's method, and it would be very helpful to have access to a bound of the type

$$
\left|\zeta\left(\frac{1}{2}+i t\right)\right| \leq C t^{1 / 6}
$$

which would be of use even if $C$ were as large as, say, 1000 .

\section{Computation}

Equation (2.1) is

$$
|S(t)| \leq \frac{2}{\pi} \log \zeta(1+\eta)+\frac{n+1}{N},
$$

where $n$ is bounded by (3.1). One can now use Lemma 3 in (3.1) to obtain a bound on $S(t)$ depending on, inter alia, the variable $r$ where $1<r \leq 2$. This general form is bloated with terms involving $\sin ^{-1} 1 / r$ and the like, and to include it here would be inexcusable. One must decide whether to use Backlund's trick (i.e. $r=2$ and twice as many zeroes) or to take a smaller value of $r$.

It can be shown, after a little computation, that the use of Backlund's trick is the better option. The general bound of (2.1) is given in an appendix, and hereafter, we shall choose $r=2$. For ease of exposition many of the error terms have been estimated — probably not optimally 6 - and the $r=2$ upper bound on (2.1) is given below in

Theorem 2. For all $t>t_{0}>3$,

$$
|S(t)| \leq a+b \log t,
$$

where

$$
a=a\left(\delta, \eta, t_{0}\right)=1.85 \log \zeta(1+\eta)+0.71 \log B(\delta)-0.58+\frac{1}{t_{0}}
$$

and

$$
b=b(\delta, \eta)=\frac{2 \theta\left(1+\frac{\pi}{3}-\sqrt{3}\right)+\left(\eta+\frac{1}{2}\right)\left(\sqrt{3}-\frac{\pi}{3}\right)}{2 \pi \log 2},
$$

where $B(\delta)$ is $\frac{3}{\delta e}$ or 2.53 according as $\theta=\frac{1}{6}+\delta$ or $\theta=\frac{1}{4}$.

Proof. The choice of $B(\delta)$ is to ensure that (4.3) holds; cf. (4.6) and (4.7).

Equation (5.3) shows that $b$ is an increasing function of both $\eta$ and $\delta$; equation (5.2) shows that $a$ is decreasing with $\eta$ and $\delta$. This inverse proportionality occurs similarly in analysis of Turing's Method [12] and it is herewith treated in like fashion.

If one wishes to investigate the size of $S(t)$ beyond some large height, then one can afford to take $\delta$ and $\eta$ smaller, so long as the term $b \log t$ in (5.1) continues to

\footnotetext{
${ }^{6}$ For example, using an upper bound $\eta \leq 1$, while true, is a weaker estimate for many of the applications. But since many of these terms are suitably small, and since Theorem 2 concisely presents the nature of the upper bound for $S(t)$, such minute savings have been ignored.
} 
dominate. Indeed, for a given $t_{0}$, the minimal value of $a+b \log t_{0}$ is sought. As an example, the Riemann hypothesis has been verified past $t_{0}=10^{10}$ (see, e.g. [13]) so it is beyond this height that explicit bounds on $S(t)$ would be of the greatest use.

As a benchmark, Rosser's bounds on $|S(t)|$ are, for $t \geq t_{0}$,

$$
|S(t)| \leq 1.588+\left\{0.137+0.443 \frac{\log \log t_{0}}{\log t_{0}}\right\} \log t
$$

The following table compares the size of $b$ - the coefficient of $\log t$ in (5.1) and the overall bound on $S(t)$, where each is obtained by Rosser's bound (5.4), Theorem 2 with $\theta=\frac{1}{4}$, and Theorem 2 with $\theta=\frac{1}{6}+\delta$.

TABLE 1. Comparison of bounds on $S(t)$

\begin{tabular}{ccccccc}
\hline \hline$t_{0}$ & \multicolumn{2}{c}{ (5.4) } & Thm 2] & $\theta=\frac{1}{4}$ & Thm 2] & $\theta=\frac{1}{6}+\delta$ \\
& $b$ & $S(t)$ & $b$ & $S(t)$ & $b$ & $S(t)$ \\
\hline $10^{10}$ & 0.1974 & 6.132 & 0.170 & 5.912 & 0.170 & 7.968 \\
$10^{12}$ & 0.1902 & 6.844 & 0.162 & 6.67 & 0.162 & 8.644 \\
$10^{14}$ & 0.1847 & 7.543 & 0.156 & 7.395 & 0.156 & 9.298 \\
$10^{16}$ & 0.1804 & 8.233 & 0.152 & 8.122 & 0.152 & 9.932 \\
$10^{18}$ & 0.1768 & 8.916 & 0.148 & 8.797 & 0.148 & 10.56 \\
$10^{20}$ & 0.1738 & 9.594 & 0.145 & 9.47 & 0.145 & 11.17 \\
$10^{40}$ & 0.159 & 16.21 & 0.131 & 15.78 & 0.126 & 17.26 \\
$10^{60}$ & 0.153 & 22.70 & 0.126 & 21.69 & 0.119 & 22.44 \\
\hline \hline
\end{tabular}

Theorem 1 follows at once from the first row of the middle column, along with the calculation of $a$ from (5.2). Note that the convexity estimates are marginally superior to Rosser's bounds in each case. Moreover, the sub-convexity estimates (the right column) improve on Rosser's bounds only in the last row. A simple computation shows that the value of $b$ obtained from the sub-convexity estimates overtakes that obtained by the middle column only when $t_{0}>10^{26}$.

Finally, note that, from [3], the bound $\zeta\left(\frac{1}{2}+i t\right) \ll t^{\theta}$, where $\theta=\frac{32}{205}$ and Theorem 2 show that

$$
|S(t)| \leq 0.1013 \log t,
$$

for $t$ sufficiently large.

\section{Conclusion}

It is tempting to see what further improvements to Theorem 1 might be possible. One way is to try to combine methods $\mathcal{A}$ and $\mathcal{B}$. That is, to take some $r<2$ and to try to replicate Backlund's trick by showing that there must be some zeroes of $f(s)$ lying on the segment left of $\frac{1}{2}+i t$, that is, the line connecting $1+\eta-r\left(\frac{1}{2}+\eta\right)+i t$ and $\frac{1}{2}+i t$. Unfortunately, such a maneuvre would require some knowledge of the nature of the horizontal distribution of the zeroes of $\Re \zeta(s)$. If such a result were known it would be natural to expect some diminution in the constants in Theorem 1 . 


\section{Appendix: the explicit bound of method $\mathcal{A}$}

For any $r \in(1,2)$, we get

$$
|S(t)| \leq \frac{2}{\pi} \log \zeta(1+\eta)+\frac{a_{1}+a_{2} \log B+a_{3} \frac{9}{2 t_{0}^{2}}+a_{4} \log \zeta(1+\eta)+a_{5} \log t}{\pi \log r},
$$

where

$$
\begin{aligned}
& a_{1}=\frac{3 \pi}{8 t_{0}}-\left(\frac{1}{2} \log 2 \pi\right) \frac{\pi}{2}-\sin ^{-1} \frac{1}{r}+r \sqrt{1-\frac{1}{r^{2}}} \\
& a_{2}=2\left(\frac{\pi}{2}-\sin ^{-1} \frac{1}{r}\right)-3 r \sqrt{1-\frac{1}{r^{2}}}+r \\
& a_{3}=r \theta\left(2-\sqrt{1-\frac{1}{r^{2}}}\right)+\sin ^{-1} \frac{1}{r}+\left(\frac{1}{2}+\eta-2 \theta\right)\left(\sin ^{-1} \frac{1}{r}-\frac{\pi}{2}+r \sqrt{1-\frac{1}{r^{2}}}\right) \\
& a_{4}=\frac{3 \pi}{2}-\sin ^{-1} \frac{1}{r}+2 r \sqrt{1-\frac{1}{r^{2}}}+1-r \\
& a_{5}=r \theta+\left(\frac{1}{2}+\eta-2 \theta\right)\left(\sin ^{-1} \frac{1}{r}-\frac{\pi}{2}+r \sqrt{1-\frac{1}{r^{2}}}\right) .
\end{aligned}
$$

The bound in (7.1) only improves on that in Theorem 1 if $\zeta\left|\left(\frac{1}{2}+i t\right)\right| \ll t^{\theta}$, where $\theta<1 / 50$.

\section{ACKNOWLEDGEMENTS}

I wish to thank Nathan $\mathrm{Ng}$ and Habiba Kadiri who suggested this problem, Roger Heath-Brown for his helpful suggestions, and the reviewer for a prompt and thorough reading of the article.

\section{REFERENCES}

1. R. J. Backlund, Über die Nullstellen der Riemannschen Zetafunction, Acta Mathematica 41 (1918), 345-375.

2. Y. F. Cheng and S. W. Graham, Explicit estimates for the Riemann zeta function, Rocky Mountain Journal of Mathematics 34 (2004), no. 4, 1261-1280. MR2095256 (2005f:11179)

3. M. N. Huxley, Exponential sums and the Riemann zeta function, $V$, Proceedings of the London Mathematical Society 90 (2005), 1-41. MR2107036 (2005h:11180)

4. H. Kadiri, Une région explicite sans zéros pour la fonction $\zeta$ de Riemann, Acta Arithmetica 117 (2005), no. 4, 303-339. MR2140161 (2005m:11159)

5. R. S. Lehman, On the distribution of zeros of the Riemann zeta-function, Proceedings of the London Mathematical Society 3 (1970), no. 20, 303-320. MR0258768 (41:3414)

6. K. S. McCurley, Explicit estimates for the error term in the prime number theorem for arithmetic progressions, Mathematics of Computation 42 (1984), no. 165, 265-285. MR726004 (85e:11065)

7. H. Rademacher, On the Phragmén-Lindelöf theorem and some applications, Mathematische Zeitschrift 72 (1959), 192-204. MR0117200 (22:7982)

8. _ Topics in analytic number theory, Die Grundlehren der mathematischen Wissenschaften, Springer-Verlag, Berlin, 1973. MR0364103 (51:358)

9. J. B. Rosser, Explicit bounds for some functions of prime numbers, American Journal of Mathematics 63 (1941), 211-232. MR0003018 (2:150e)

10. Y. Saouter and P. Demichel, A sharp region where $\pi(x)-l i(x)$ is positive, Mathematics of Computation 79 (2010), no. 272, 2395-2405. MR2684372

11. E. C. Titchmarsh, The theory of the Riemann zeta-function, 2nd ed., Oxford Science Publications, Oxford University Press, Oxford, 1986. MR882550 (88c:11049)

12. T.S. Trudgian, Improvements to Turing's Method, Mathematics of Computation (to appear).

13. S. Wedeniwski, Results connected with the first 100 billion zeros of the Riemann zeta function, http://www.zetagrid.net/zeta/math/zeta.result.100billion.zeros.html, 2004. 
Department of Mathematics and Computer Science, University of Lethbridge, Alberta, Canada, T1K 3M4

E-mail address: tim.trudgian@uleth.ca 